\title{
可读性增强的流图生成
}

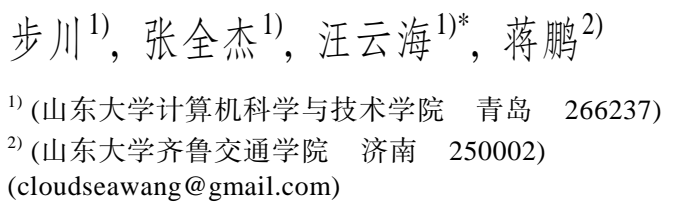

摘 要：为了增强流图可读性, 帮助用户更高效地识别染色层, 提出一种基于流图已有布局, 优化流图中染色层的 颜色分配与标签布局的方法. 首先根据流图的布局数据建立其颜色分配评价函数, 通过遗传算法进行颜色分配方案 优化, 最大化染色层之间的颜色差异和染色层与背景的亮度差异; 然后在每个染色层上运行滑动窗口, 通过最小二 乘法拟合自动调整标签倾斜角度以适应染色层形状变化, 得到标签尺寸自适应的标签布局结果. 通过用户测试与定 量评估, 将文中方法与现有方法在多个真实和模拟数据集上进行实验的结果表明, 该方法能够显著提高染色层的识 别正确率, 降低识别耗时, 同时增大标签尺寸, 从而有效地提高流图可读性.

关键词: 流图; 颜色分配; 标签布局

中图法分类号: TP391.41 DOI: 10.3724/SP.J.1089.2021.18688

\section{Streamgraph Generation with Enhanced Readability}

\author{
Bu Chuan $^{1)}$, Zhang Quanjie ${ }^{1)}$, Wang Yunhai ${ }^{1)^{*}}$, and Jiang Peng ${ }^{2)}$ \\ ${ }^{1)}$ (School of Computer Science and Technology, Shandong University, Qingdao 266237) \\ ${ }^{2)}$ (School of Qilu Transportation, Shandong University, Jinan 250002)
}

\begin{abstract}
In order to enhance the readability of the streamgraph and assist users identify the target layer more efficiently, a method to optimize the color assignment and label layout in streamgraph based on the existing layout of streamgraph is proposed. First, the color assignment evaluation function of the streamgraph is established according to the layout, and then the color assignment result is got which can maximize the color difference between layers as well as the luminance difference between the background color and layers through genetic algorithm. Secondly, the sliding window is used to traverse each layer, and the tilt angle of each label is automatically adjusted by least squares to fit the shape change of each layer. Then the label layout results are obtained with adaptive label size. The method is compared with existing methods on multiple real and simulated datasets through user study and quantitative evaluation. The results show that the method can significantly improve the recognition accuracy, reduce the recognition time of the layers, and increase the label size at the same time, which means it can improve the readability of the streamgraph effectively.
\end{abstract}

Key words: streamgraph; color assignment; label layout

收稿日期：2020-09-29; 修回日期：2020-12-15. 基金项目：国家自然科学基金(61772315); 国家自然科学基金中德合作研究项目 (61861136012). 步川(1995一), 男, 硕士研究生, 主要研究方向为信息可视化; 张全杰(1997一), 男, 硕士研究生, 主要研究方向为信 息可视化; 汪云海(1984一), 男, 博士, 教授, 博士生导师, 论文通讯作者, 主要研究方向为数据可视化、人机交互; 蒋鹏(1986一), 男, 博士, 副教授, 主要研究方向为计算机视觉、图像处理、人工智能. 
堆叠面积图是一种常用的展示多时间序列数 据的图表形式, 所有时间序列的数值大小体现为 图上对应染色层在坚直方向上的厚度, 将这些染 色层紧密堆叠在一起能够有效地展示时间序列之 间以及整体的相对数值大小. 而流图是堆叠面积 图的一个变种, 它拥有可以进行弯曲的最底部基 线, 通过调整基线的形状, 可以使流图中染色层变 得更加平滑. 流图自面世以来便受到欢迎, 被广泛 应用于社交媒体、电影票房等数据的可视化展示.

流图有基线形状、染色层排序、标签布局和颜 色分配 4 个要素 ${ }^{[1]}$, 如何通过它们增强其可读性一 直是流图研究的热门课题. 当前的工作 ${ }^{[1-3]}$ 多通过 优化流图的基线形状和染色层排序让流图上的染 色层更加平滑, 方便用户读取流图的数据信息, 进 而增强可读性. 也有一些工作 ${ }^{[4-6]}$ 从交互设计的角 度人手, 通过数据查询、建立层次结构等方法辅助 用户在流图上进行探索, 进而增强流图可读性. 尽 管当前工作在流图可读性增强上取得了一定进展, 且得到一些不错的效果, 但如何通过颜色分配与 标签布局增强流图可读性, 仍然是一个值得深人 研究的问题.

本文提出优化流图颜色分配和标签布局的方 法来增强流图可读性. 首先建立流图的颜色分配 方案评价函数，其中考虑了流图的布局数据、时间 序列之间的颜色差异和时间序列与背景颜色的亮 度差异，通过遗传算法优化颜色分配方案得到最 终结果; 其次在每个时间序列上运行滑动窗口, 通 过最小二乘法拟合自动调整标签倾斜角度增大标 签尺寸得到标签布局结果.

为了验证本文方法有效性，通过用户测试和 定量评估，将该方法与现有方法在真实和模拟数 据集上进行对比, 结果表明, 该方法能够有效地增 强流图可读性.

\section{1 相关工作}

\section{1 流图可读性增强}

流图的概念最初由 ThemeRiver ${ }^{[3]}$ 引出, 它通 过调整基线使流图整体上下对称. 随后, Byron 等 ${ }^{[1]}$ 正式介绍了流图, 详细描述了流图背后的数学原 理和设计考量，通过优化基线形状尽可能减少流 图中染色层的上下波动, 使染色层看起来更加自 然和平滑，进而增强了流图可读性；同时，根据染 色层的出现时间对染色层进行排序, 得到了不错 的效果，但是这种排序方法只适用于特定类型的
数据. Bartolomeo 等 ${ }^{[2]}$ 提出一种更适用于一般类型 数据的流图排序方法, 通过迭代的方式优化排序, 将厚度变化剧烈的染色层尽可能地放置在流图的 外侧, 平滑的染色层放置在流图内侧, 大大增强了 流图的可读性; 同时, 提出一种基于1-范数的基线 计算方法, 能够有效地避免染色层的突元波动.

除了布局优化，流图的交互设计也被用来增 强可读性. TouchWave $\mathrm{e}^{[4]}$ 将交互式布局调整和数据 查询引人流图, 旨在解决流图感知问题和提高可 读性. Thudt 等 $^{\left[{ }^{5}\right]}$ 提出一个交互式的流图基线校直 方案, 以提高所选层的可读性; 点击一个单独的染 色层, 该染色层的一侧边界会变为水平, 这样可以 很容易地观察到染色层的厚度. MultiStream ${ }^{[6]}$ 将流 $^{-}$ 图中时间序列组织成层次结构，同时利用焦点+ 上下文技术，允许用户在不同粒度上对流图进行 探索.

\section{2 颜色编码}

颜色是可视化中图形元素的基本属性之一 ${ }^{[7]}$. 为了生成富有表现力的可视化作品, 对颜色编码进 行精心设计十分重要. 颜色编码工作分为 2 部分: (1) 调色板生成(如 ColorBrewer ${ }^{[8]}$, Colorgorical ${ }^{[9]}$ ); (2) 颜色分配. 将颜色对应到具体的图形元素上. 本文使用现有的调色板在流图上进行自动颜色分 配, 以增强流图可读性.

颜色分配需要考虑的因素有颜色和谐 ${ }^{[10]}$ 、类可 见度 ${ }^{[11]}$ 和感知距离 ${ }^{[12]}$ 等. Hurter 等 ${ }^{[13]}$ 通过增强邻近 线路的颜色差异给地铁线路图染色; Wang 等 ${ }^{[14]}$ 通 过增强散点图上不同类之间的颜色差异和类与背 景的亮度差异提高类区分度. Kim 等 ${ }^{[15]}$ 提出一种感 知驱动的方法, 融合颜色美学和对比度对无序图 像片段进行颜色分配. 在流图上, Byron 等 ${ }^{[1]}$ 根据 时间序列的数据特征, 如数值大小、开始时间等进 行颜色编码，向用户传递更多的信息; 为了体现层 次结构信息, MultiStream ${ }^{[6]}$ 将兄弟时间序列对应的 染色层分配相近的颜色. 但是在一般的流图中，时 间序列之间的数据特征差异并不显著，同时层次 结构信息也较少存在, 因此上述 2 种方法不适用于 一般的流图颜色分配. 本文通过增大流图中染色 层的颜色差异和染色层与背景的亮度差异, 提高 染色层区分度，进而增强流图可读性.

\section{3 标签布局}

标签布局是自动制图领域的核心问题之一 ${ }^{[16]}$. 良好的标签布局有助于图的阅读和美感提升 ${ }^{[17]}$, 但这一问题也被证明是一个 NP 难问题 ${ }^{[18]}$. 标签布 局通常基于点特征进行布局算法的设计 ${ }^{[19-21]}$, 也 
有一些算法基于线、面特征进行布局算法设计. Yamamoto 等 ${ }^{[21]}$ 提出一种网格算法, 面向线、面特 征进行标签布局. Pokonieczny 等 ${ }^{[22]}$ 使用神经网络 在地图中对多边形进行标签布局. 因为在流图上进 行标签布局需要将标签严格放置在染色层内部 ${ }^{[1]}$, 所以本文基于流图的面积特征进行标签布局.

标签是流图的要素之一，在图上添加标签能 够帮助用户跳过查询图例的步骤, 更高效率地寻 找到目标染色层. Byron 等 ${ }^{[1]}$ 使用简单的蛮力算法 进行标签布局以最大化标签尺寸. Bartolomeo 等 ${ }^{[2]}$ 在染色层内部水平放置标签, 同时使用滑动窗口 方法寻找可以将标签尺寸最大化的放置位置, 虽 然提升了运算效率，但是造成染色层内部空间的 浪费，标签尺寸较小。本文在使用滑动窗口的同 时，使用最小二乘法拟合自动调整标签角度，可以 更有效地利用染色层内部空间，增大标签尺寸。

\section{2 本文方法}

\section{1 问题定义}

本文方法分为颜色分配与标签布局 2 部分. 其 输人如下.

(1) 流图 $G$, 如图 1 所示. $G$ 的数据是离散的, 包含 $n$ 个时间序列，表示为 $f_{i}, i \in[1, n]$ ，每个时间 序列中包含 $m$ 个非负值. 流图上每个染色层对应 一个时间序列，形状由其上下边界决定，相邻的染 色层共用边界. 流图中共有 $n+1$ 条边界, 表示为 $g_{i}, i \in[0, n]$, 每个边界均是一个点集, 其中包含 $m$ 个点.

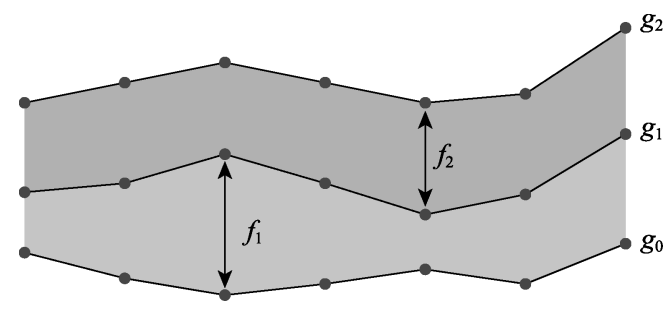

图 1 流图示意图

（2）调色板 $P$ 和背景颜色 $C_{\mathrm{b}} . P$ 中包含 $q$ 个 颜色, 表示为 $p_{i}, i \in[1, q]$.

（3）标签集合 $L . L$ 中包含 $n$ 个标签, 表示为 $l_{i}, i \in[1, q] . l_{i}$ 与 $f_{i}$ 一一对应.

输出结果如下.

(1) 颜色分配方案: $\tau:\left[C_{1}, C_{2}, \cdots, C_{n}\right]$. 其中, $\tau$ 为 $G \rightarrow P$ 的映射, $C_{i}$ 为时间序列 $f_{i}$ 对应染色层
的颜色, $C_{i} \in P$.

（2）标签布局方案. $L$ 中所有标签均有放置位 置和倾斜角度, 每个标签都在对应染色层的内部.

\section{2 颜色分配}

通过优化颜色分配增强流图流图可读性，需 要考虑 2 个方面：(1) 增大染色层的颜色差异以便 用户识别不同染色层; (2) 增大流图整体与背景颜 色的对比, 凸显流图的轮廓, 方便用户观察流图的 整体形状. 定义颜色分配目标函数为

$$
\arg \max _{\tau} E(\tau)=\sum_{i=1}^{n} \sum_{j=1}^{n} E_{\mathrm{d}}\left(C_{i}, C_{j}\right)+\sum_{i=1}^{n} E_{\mathrm{l}}\left(C_{i}, C_{\mathrm{b}}\right)
$$

其中， $E_{\mathrm{d}}$ 表示染色层之间的颜色差异； $E_{1}$ 表示染 色层与背景的亮度差异; $C_{i}$ 表示 $f_{i}$ 对应染色层的 颜色. 通过最大化 $E(\tau)$ 得到最终的颜色分配方案.

\subsection{1 染色层之间的颜色差异}

区分度在人眼显著性检测中起到关键作用 ${ }^{[23]}$, 指一个物体可以从环境中区分出来的程度. 为了 方便用户识别染色层, 需要增大染色层之间的区 分度. 在区分度评估时, Margolin 等 ${ }^{[24]}$ 即是通过图 片上不同区域的颜色差异进行评价. 本文也通过 增大染色层之间的颜色差异增大染色层的区分度， 通过 CIEDE2000 色差公式计算颜色差异,即

$$
E_{\mathrm{d}}\left(C_{i}, C_{j}\right)=w_{i j} \cdot D\left(C_{i}, C_{j}\right)
$$

其中, $D\left(C_{i}, C_{j}\right)$ 为颜色 $C_{i}$ 和 $C_{j}$ 的颜色差异; $w_{i j}$ 为在计算染色层 $f_{i}$ 和 $f_{j}$ 对应染色层的颜色差异时 需要考虑的权重.

\subsection{2 染色层与背景的亮度差异}

在增强染色层之间区分度的同时，也需要考 虑流图整体与背景颜色的区分度

$$
E_{\mathrm{l}}\left(C_{i}, C_{\mathrm{b}}\right)=w_{i j} \cdot L\left(C_{i}, C_{\mathrm{b}}\right)
$$

其中, $L\left(C_{i}, C_{\mathrm{b}}\right)$ 为颜色 $C_{i}$ 和 $C_{\mathrm{b}}$ 的亮度差异, 权重 $w_{i j}$ 与式(2)相同. 当前背景颜色一般选择白色或其 他浅色, 可以通过增强染色层与背景的亮度差异 增强流图整体的区分度, 如图 2 所示. 与图 $2 \mathrm{~b}$ 相 比，图 2c 中流图的轮廓更易识别.

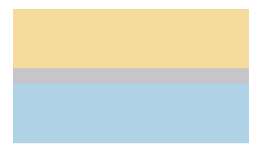

a. 原始图

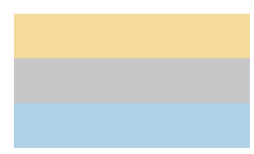

b. 增大灰层厚度

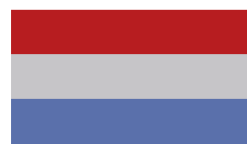

c. 增大亮度差异
图 2 颜色分配目标函数示意图

\subsection{3 权 重}

对于染色层 $f_{i}$ 和 $f_{j}$, 权重 $w_{i j}$ 分为 2 部分, 即 


$$
w_{i j}=N\left(f_{i}, f_{j}\right) \cdot T\left(f_{i}, f_{j}\right)
$$

其中, $N\left(f_{i}, f_{j}\right)$ 为 2 个染色层的相邻边界距离, 使 相邻染色层的颜色差异更大; $T\left(f_{i}, f_{j}\right)$ 为 2 个染色 层的厚度权重. 如图 2 所示, 与图 2b 相比, 图 2a 中的灰色染色层厚度薄, 更加不容易被用户识别, 因此在计算颜色差异度时, 需要对薄的染色层施 加额外的注意力. 厚度权重可以取 2 个染色层厚度 数据的算数平均值、中值、最小值等的倒数, 效果 是让一些较薄的染色层更加容易被识别出来. 实 验结果表明, 取最小值的倒数效果最好.

\subsection{4 遗传算法}

图 $G$ 中有 $n$ 个染色层, 调色板 $P$ 中有 $q$ 个颜 色, 对应的有 $n^{q}$ 个颜色分配方案, 因此, 遍历所有 颜色分配方案以获得最优解的方法是不可取的. 如果流图中时间序列 $f_{i}$ 是有序的, 那么颜色分配 方案 $\tau:\left[C_{1}, C_{2}, \cdots, C_{n}\right]$ 可以视为一个颜色的序列. 使用遗传算法将颜色分配方案 $\tau$ 视为个体的基因 序列, 通过一代代选择、变异、遗传得到一个可行 的颜色分配方案. 相关参数设置如下: 种群总量为 50 , 复制比例为 $20 \%$, 变异概率为 0.01 . 具体步骤 如下.

输人. 流图 $G$, 调色板 $P$, 背景颜色 $C_{\mathrm{b}}$.

输出. 颜色分配方案 $\tau$.

Step1. 初始化种群. 对于种群中的每个个体 $\tau$, 首 先对 $P$ 进行洗牌操作, 然后循环读取 $P$, 从中逐个取出 颜色填人 $\tau$, 直至 $\tau$ 中颜色个数为 $n$.

Step2. 使用式(1)计算种群中所有个体的适应度.

Step3. 检查是否满足 2 个迭代终止条件之一: (1) 迭代次数达到 300; (2) 最大适应度连续 30 次迭代没有 提升. 若满足, 返回当前种群中适应度最高的个体; 否 则, 执行下一步.

Step4. 通过复制、交叉、变异方式生成下一代种群:

Step4.1. 复制. 将本代种群中适应度排名前 $20 \%$ 的个体直接复制给下一代;

Step4.2. 交叉. 使用轮盘赌方法, 每次选出本代 种群里 2 个适应度较高的个体作为父母, 通过交换一段 基因序列得到下一代个体;

Step4.3. 变异. 除复制得到的个体外, 在一定概 率下随机交换个体的基因序列中的 2 个基因;

Step4.4. 得到下一代种群, 转 Step2.

需要说明的是, 在 Step4.2 中, 为了避免得到 含有较多重复基因的下一代个体, 采用基因交叉 方式 ${ }^{[14]}$, 即在交换基因片段之前对个体基因序列 进行预处理, 将即将发生重复的基因剔除. 大量实 验结果显示, 算法一般会在迭代 100 次左右时返回
最终结果, 算法过程如图 3 所示.

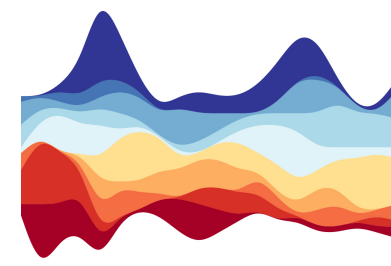

a. 初始化

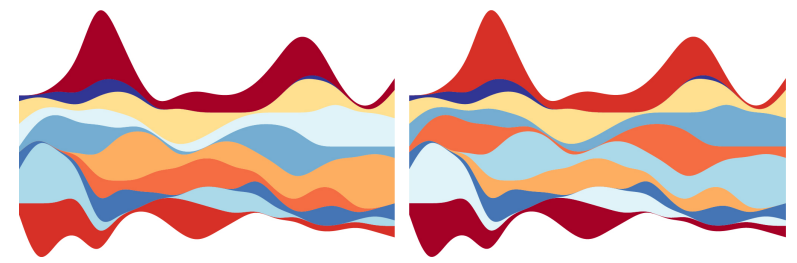

c. 迭代 80 次

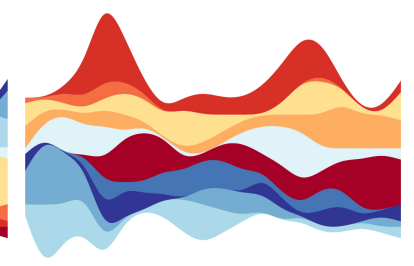

b. 迭代 10 次
图 3 遗传算法过程示意图

更多的颜色分配前后对比结果如图 4所示, 其 中, 所有流图的染色层数为 8 13; 奇数行为初始 颜色分配结果, 偶数行为本文颜色分配方法的结 果; 奇数行与偶数行的图片一一对应.

\subsection{5 参数分析}

(1) 染色层与背景颜色的亮度差异

式(1)分为染色层之间的颜色差异 $E_{\mathrm{d}}$ 和染色层 与背景颜色的亮度差异 $E_{1} 2$ 个部分. 图 5 所示为 在式(1)中是否考虑 $E_{1}$ 的对比示意图.

图 5a 中, 顶部染色层为浅黄色, 与背景颜色 亮度差异小, 较容易产生混淆; 图 5b 中, 顶部染 色层为红色, 与背景颜色亮度差异大, 对比明显. 与图 5b 相比, 图 5a 的顶部轮廓更加不容易被用户 识别, 可读性较低. 所以有必要在式(1)中考虑亮 度差异 $E_{1}$.

\section{(2) 厚度权重}

式(1)中的 $w_{i j}$ 分为 2 部分: 染色层之间的相邻 边界距离 $N\left(f_{i}, f_{j}\right)$ 与染色层的厚度权重 $T\left(f_{i}, f_{j}\right)$. 图 6 所示为在式(1)中是否考虑 $w_{i j}$ 的对比示意图.

若只考虑染色层之间的相邻距离, 会使厚度 较小且与相邻染色层的边界距离较短的染色层较 难识别, 这主要是由于该染色层与相邻染色层颜 色差异较小. 如图 6 中红色箭头指向的目标染色层 所示, 图 6a 中的目标染色层较难识别出来; 而图 $6 \mathrm{~b}$ 中因为考虑了 $w_{i j}$, 导致在计算目标染色层与其 相邻染色层的颜色差异时考虑的权重变大, 颜色 差异变得明显从而更易识别, 避免了如图 6a 所示 的情况. 


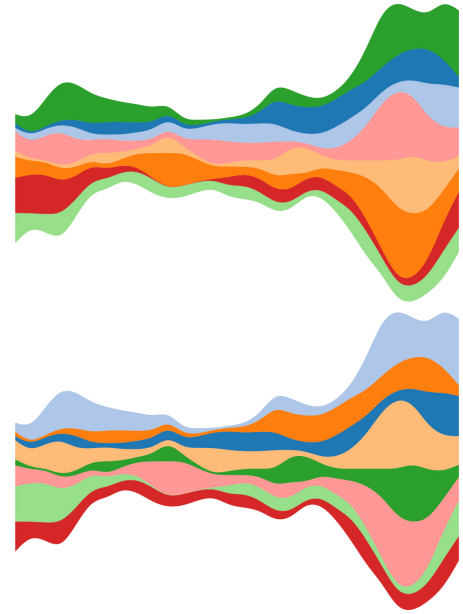

a. 8 层流图

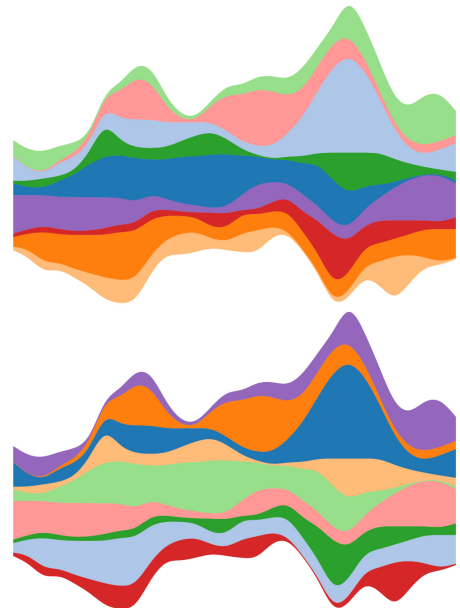

d. 9 层流图

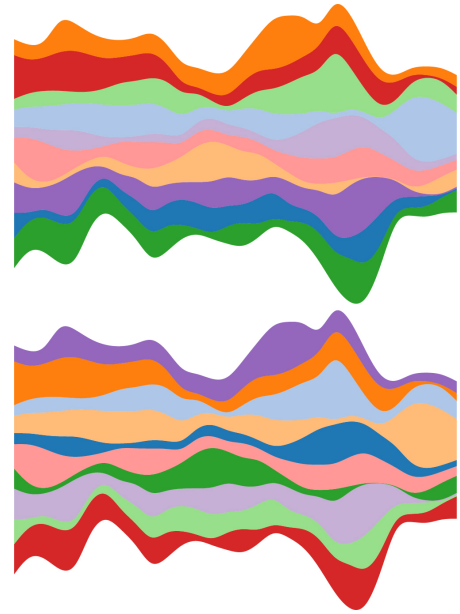

b. 10 层流图

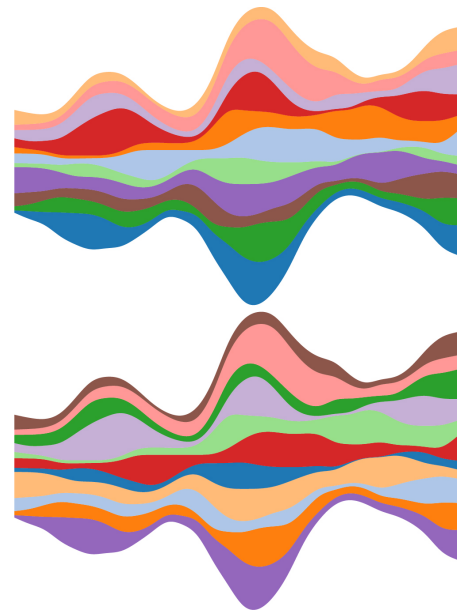

e. 11 层流图

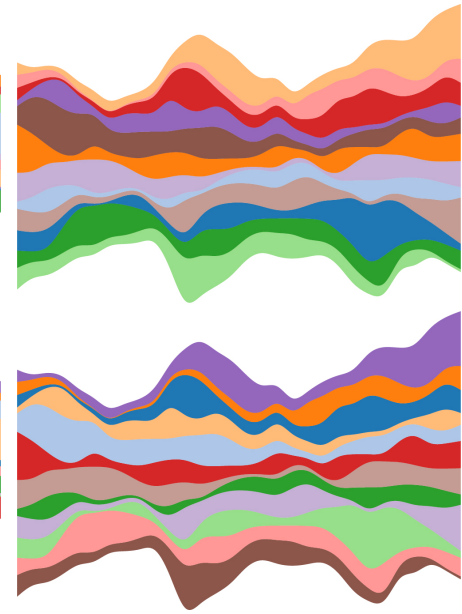

c. 12 层流图

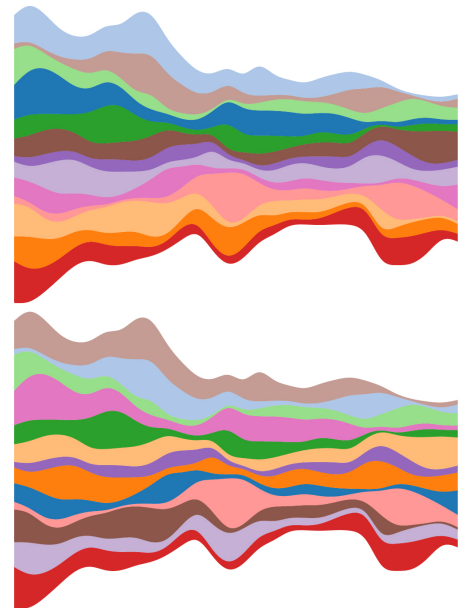

f. 13 层流图

图 4 颜色分配前后对比

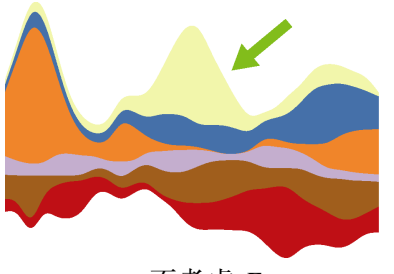

a. 不考虑 $E_{1}$

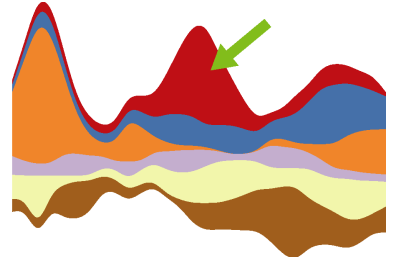

b. 考虑 $E_{1}$
图 5 式(1)中是否考虑 $E_{1}$ 对比图

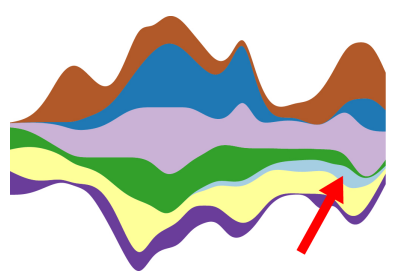

a. 不考虑 $w_{i j}$

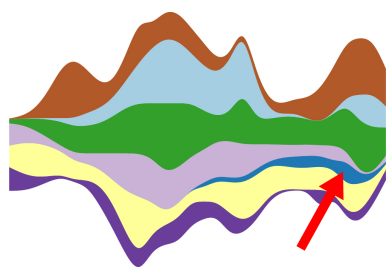

b. 考虑 $w_{i j}$
图 6 式(1)中是否考虑 $w_{i j}$ 对比图

\section{3 标签布局}

使用流图完成任务的一个重要步骤是找到任 务所需时间序列对应的染色层 ${ }^{[1]}$. 在流图上直接放
置标签可以避免染色层过多导致图例颜色不够用 的问题 ${ }^{[1]}$, 同时也可以让用户跳过查询图例的步 骤, 直接在流图上寻找所需染色层. 更大尺寸的标 签能够让用户更快识别出其所在的染色层, 进而 增强流图可读性.

在染色层上放置标签需要标签严格位于染色 层的内部 ${ }^{[1]}$, 不能与其他染色层重叠. Bartolomeo 等 ${ }^{[2]}$ 在染色层内部水平放置标签, 但是流图中的染 色层一般是上下起伏的, 水平放置标签会造成空 间的浪费，如图 7 所示. 如果给标签一个倾斜的角 度, 可以大大增加标签的尺寸, 更有效地利用染色 层内部空间.

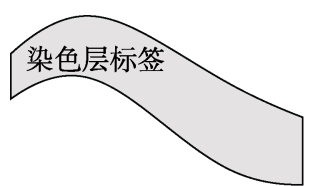

a. 水平放置标签

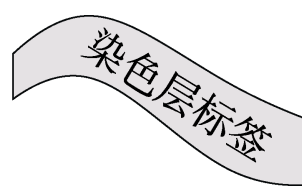

b. 倾斜放置标签
图 7 不同标签放置方式对比 
染色层的边界由一系列的点连接而成, 这些 点分为上、下边界点集 $t$ 和 $b$. 本文在染色层上运 行滑动窗口算法, 对进人窗口的点集通过最小二 乘法进行拟合，得到标签的倾斜角度; 同时，为了 避免标签倾斜角度过大，将标签最大倾斜角度设 为 $\alpha_{\max }=45^{\circ}$ [25]. 算法步骤如下.

输人. 染色层的上边界 $t$, 下边界 $b$, 标签的 宽高比 $r$, 最大宽度 $w_{\text {max }}$, 最小宽度 $w_{\text {min }}$, 最大倾 斜角度 $\alpha_{\text {max }}$, 标签缩小比例 $s=0.9$.

输出. 标签的尺寸、中心位置、倾斜角度.

Step1. 初始化. 令标签宽度 $w=w_{\text {max }}$, 高度 $h=w / r$.

Step2. 令滑动窗口宽度为 $w$ (图 8 中红色线段长 度), 其中心横坐标为 $x_{\text {center }}$, 在染色层上自左向右移动 滑动窗口, 每次令 $x_{\text {center }}$ 向右移动一个单位, 不断尝试 进行标签布局:

Step2.1. 上下边界 $t, b$ 均为点集, 可以得到 $t, b$ 上位于以 $x_{\text {center }}$ 为中心, 宽度为 $w$ 的范围内的点集 $Q_{\text {initial }}$, 如图 8a 中绿色点集所示;

Step2.2. 对 $Q_{\text {initial }}$ 使用最小二乘法进行拟合，结 果如图 8b 所示. 得到拟合直线 $L_{\mathrm{fit}}$, 对应的标签中心 $C\left(x_{\text {center }}, y_{\text {center }}\right)$, 标签倾斜角度 $\alpha$ (即 $L_{\text {fit }}$ 与水平方向的 夹角), 若 $|\alpha|>\alpha_{\text {max }}$, 则令 $\alpha=\operatorname{sgn}(\alpha) \cdot \alpha_{\text {max }}$ ；

Step2.3. 将染色层以 $C$ 为中心旋转 $\alpha$, 如图 8c 所示, 得到对应标签宽度 $w$ 的上边界点集 $Q_{\mathrm{top}}$ 和下边界 点集 $Q_{\mathrm{bot}}$;

Step2.4. 计算 $Q_{\mathrm{top}}$ 和 $Q_{\mathrm{bot}}$ 的最小高度差 $H_{\mathrm{d}}$, 如 图 8c 所示. 若 $H_{\mathrm{d}} \geqslant h$, 则保存当前标签尺寸 $w, h$, 标签 中心 $C$ 和倾斜角度 $\alpha$ ，执行下一步; 否则，继续向右移 动滑动窗口, 转 Step2.1, 直至滑动窗口移动至染色层最 右端.

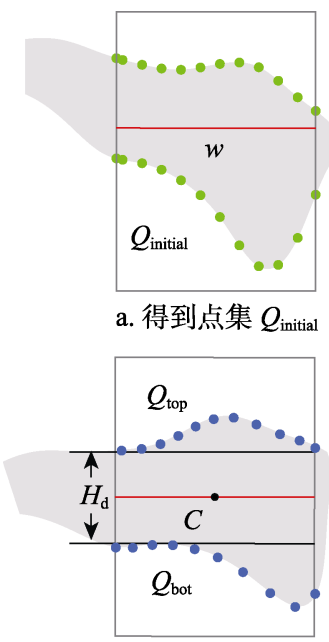

c. 验证是否合法

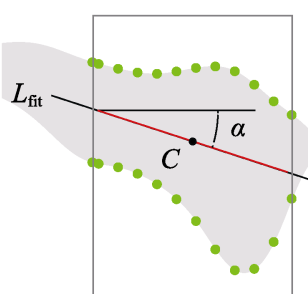

b. 进行拟合

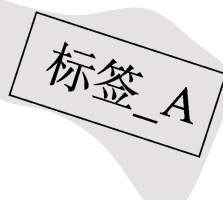

d. 得到结果
图 8 标签布局算法流程示意图
Step3. 若得到标签放置结果, 则直接返回标签放 置的横坐标 $x_{\text {center }}$, 宽度 $w$, 倾斜角度 $\alpha$; 否则, 缩小标 签尺寸, 令 $w=\min (w \cdot s, w-1), h=w / r$.

Step4. 若 $w \geqslant w_{\text {min }}$, 转 Step2; 否则, 令 $w=0$ 并直 接结束算法.

需要说明的是, 如果不能得到一个布局位置 使标签尺寸大于最小尺寸, 则不在流图上放置这 个标签. 本文标签布局整体效果如图 9 所示.

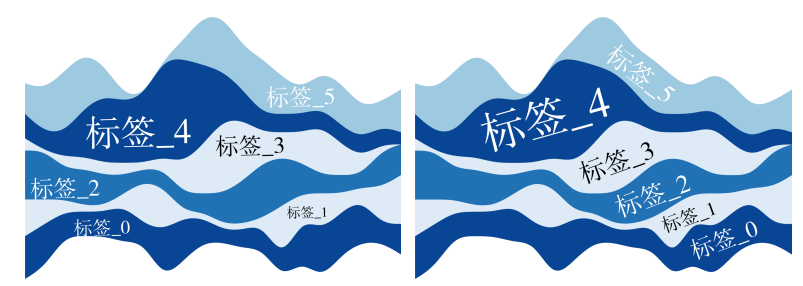

a. 水平标签布局

b. 本文标签布局

图 9 标签布局结果对比

\section{3 算法评估}

对本文提出的颜色分配和标签布局进行算法 评估. 所有流图数据均首先使用 Bartolomeo 等 ${ }^{[2]}$ 的流图布局算法进行布局，在此基础上进行颜色 分配与标签布局的工作.

\section{1 颜色分配算法评估}

通过用户测试评估本文颜色分配算法对增强 流图可读性的有效性. 其中, 将背景颜色设置为白 色, 调色板采用 Tableau 软件自带调色板 Tableau20.

(1) 数据集. 使用的数据由 2 个真实数据集和 8 个随机数据组成. 2 个真实数据集来自 Thudt 等 ${ }^{[5]}$ 使用的 311 电话数据集和电影票房数据集, 分别有 10 个和 30 个染色层; 随机数据的染色层个数为 16 30. 对每个数据分别进行随机颜色分配和使用 本文算法进行颜色分配, 最终的流图-颜色分配组 合共有 20 个.

(2) 参与者. 20 位志愿者参与了用户测试, 其 中, 18 男 2 女, 年龄范围为 18 26 岁, 均无流图设 计经验, 且均无视觉障碍.

（3）任务设计. 为了评估本文颜色分配算法对 流图可读性的影响, 用户需要根据给定的一个颜 色，在流图中寻找具有相同颜色的染色层，用户的 正确率和完成时间会被记录下来. 需要注意的是, 由于调色板中颜色有限, 因此流图中可能有不同 的染色层具有相同的颜色. 在这种情况下, 用户只 需要选中其中一个染色层即可被认定为正确.

(4) 任务流程. 所有的用户测试均在线上进 
行, 用户被要求只能够使用鼠标进行操作，不能够 使用其他工具辅助作答. 在进行正式任务之前, 用 户先进行 2 个训练任务以熟悉基本操作; 同时, 为 了避免相同流图的不同颜色分配会对用户的作答 造成影响, 本文通过拉丁方组织流图数据的展示 顺序.

(5) 结果分析. 用户测试结果如表 1 和表 2 所 示. 从表 1 可以看出, 本文颜色分配算法高于随机 颜色分配算法，且配对 $t$ 检验的 $p$ 值为 $5.3 \times 10^{-4}$, 远小于 0.05. 从表 2 可以看出, 本文颜色分配算法 的时间少于随机颜色分配算法的时间，且配对 $t$ 检 验的 $p$ 值为 $2.5 \times 10^{-6}$, 远小于 0.05 . 因此, 使用本 文算法进行颜色分配能够显著地提高用户完成任 务的准确率, 缩短所用时间, 即增强流图可读性.

表 1 用户测试正确率结果

\begin{tabular}{ccc}
\hline 算法 & 正确率均值 & 标准差 \\
\hline 本文 & 0.96 & 0.049 \\
随机 $^{[1]}$ & 0.87 & 0.114 \\
\hline
\end{tabular}

表 2 用户测试时间结果

\begin{tabular}{ccc}
\hline 算法 & 时间均值/s & 标准差 \\
\hline 本文 & 3645.3 & 1066.6 \\
随机 $^{[1]}$ & 4904.7 & 1471.7 \\
\hline
\end{tabular}

\section{2 标签布局算法评估}

\subsection{1 时间复杂度}

在每个染色层上运行滑动窗口，其时间复杂 度为 $O(n m)$, 对进入滑动窗口的点集使用最小二 乘法进行计算, 其时间复杂度为 $O(m)$, 尺寸每次 等比缩小 $s$, 最终的算法复杂度为 $O\left(\mathrm{~nm}^{2} \log (s)\right)$.

\subsection{2 定量评估}

（1）实验设计. 对每个流图分别使用本文标签 布局算法与 Bartolomeo 等 ${ }^{[2]}$ 的水平标签布局算法 进行标签布局并计算所有标签所占面积的和，然 后计算 2 个面积和的比值. 通过分析该比值数据, 来评估本文算法对增强流图可读性的有效性.

(2) 数据生成. 随机生成 1000 份流图数据, 时间序列个数 4 34 不等, 每个时间序列对应标签 的字符个数 2 12 不等.

(3) 对比结果. 实验结果如图 10 所示, 可以看 出, 流图中时间序列个数越多, 本文标签布局算法 对于标签尺寸的增大效果越强. 原因是随着染色 层个数增多, 单个染色层的相对厚度变小, 其形状 的上下波动增强, 水平放置标签的方式浪费染色 层内部空间增大. 综上可知, 本文标签布局算法能 够有效地增大标签尺寸，增强流图可读性.

一些标签布局算法的结果如图 11 和图 12 所示, 其中, 图 11 中的流图染色层数为偶数, 图 12 中的

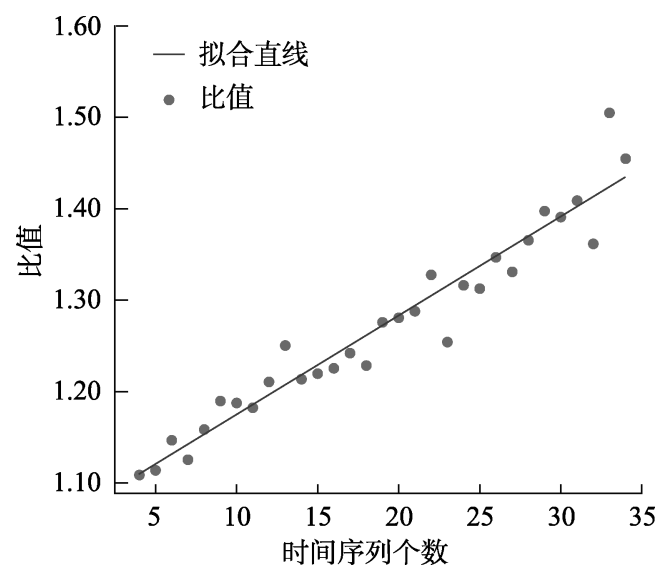

图 10 不同时间序列个数的面积比值

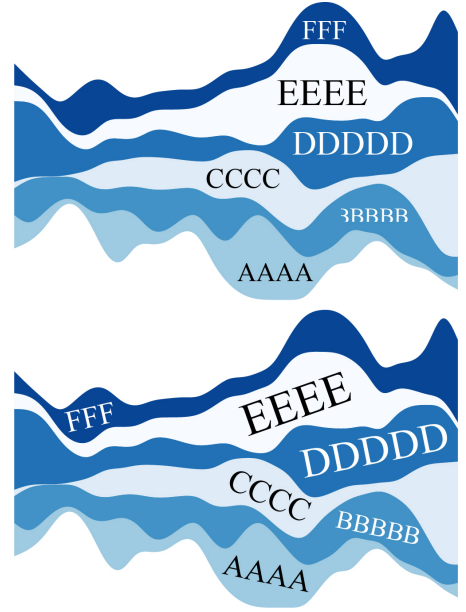

a. 6 层流图

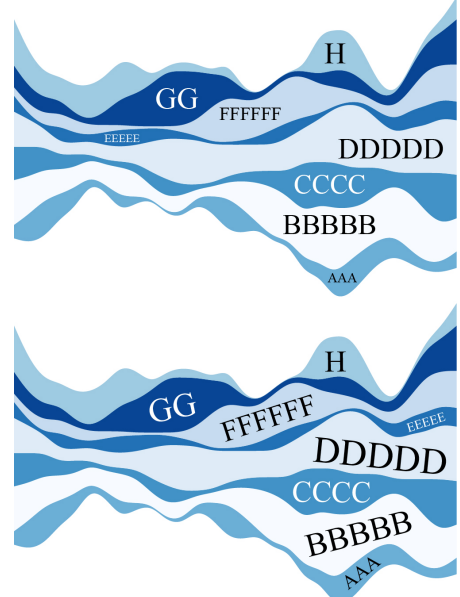

b. 8 层流图

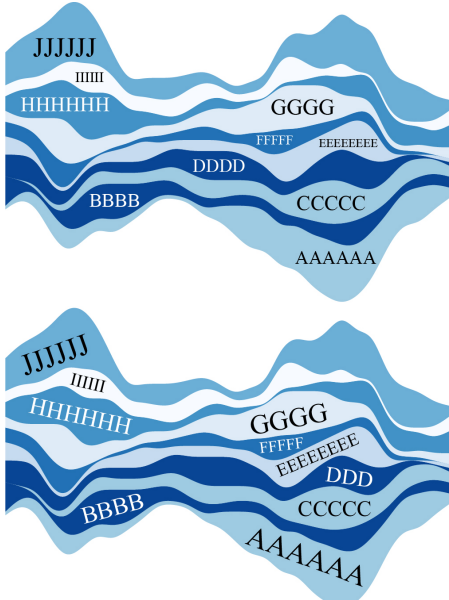

c. 10 层流图

图 11 染色层数为偶数时不同标签布局结果对比 


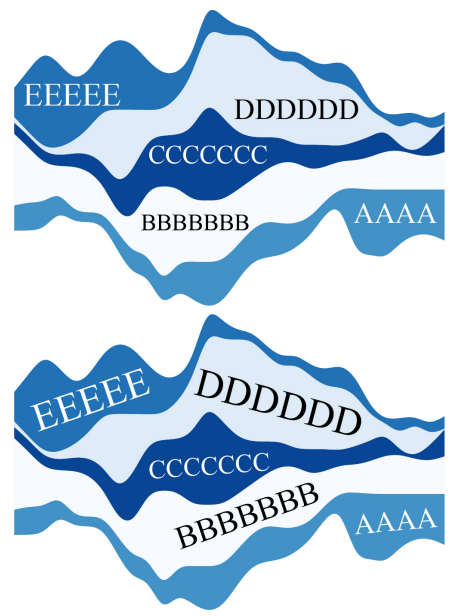

a. 5 层流图

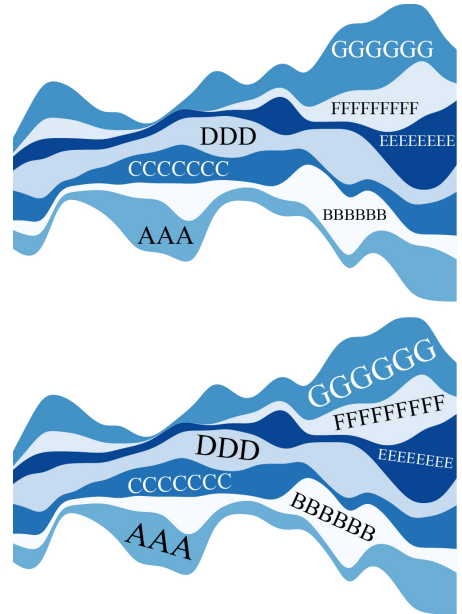

b. 7 层流图

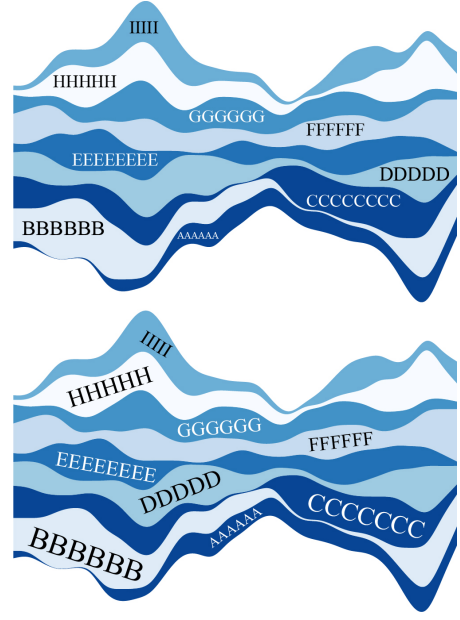

c. 9 层流图

图 12 染色层数为奇数时不同标签布局结果对比

流图染色层数为奇数; 图中第 1 行为水平标签布局 算法的结果, 第 2 行为本文标签布局算法的结果, 2 行的图片上下一一对应.

\section{4 结 语}

本文在流图已有布局的基础上，通过优化颜 色分配与标签布局增强流图可读性. 首先建立流 图的颜色分配评价函数, 通过遗传算法迭代获得 能够最大化染色层之间的颜色差异和染色层与背 景颜色的亮度差异的颜色分配结果; 其次在染色 层上运行滑动窗口, 使用最小二乘法自动调整标 签倾斜角度，增大了标签尺寸. 定量评估和用户测 试结果表明，本文方法能够有效地增强流图可读 性. 虽然本文标签布局算法能够有效地增大标签 尺寸，但是运算效率不够高效，不足以支持实时交 互. 在未来的工作中, 需继续简化运算复杂度, 提 高交互效率.

\section{参考文献(References):}

[1] Byron L, Wattenberg M. Stacked graphs-geometry \& aesthetics[J]. IEEE Transactions on Visualization and Computer Graphics, 2008, 14(6): 1245-1252

[2] Bartolomeo M D, Hu Y F. There is more to streamgraphs than movies: better aesthetics via ordering and lassoing[J]. Computer Graphics Forum, 2016, 35(3): 341-350

[3] Havre S, Hetzler E, Whitney P, et al. ThemeRiver: visualizing thematic changes in large document collections[J]. IEEE Transactions on Visualization and Computer Graphics, 2002, 8(1): 9-20

[4] Baur D, Lee B, Carpendale S. TouchWave: kinetic multi-touch manipulation for hierarchical stacked graphs[C] //Proceedings of the ACM International Conference on Interactive Tabletops and Surfaces. New York: ACM Press, 2012: 255-264

[5] Thudt A, Walny J, Perin C, et al. Assessing the readability of stacked graphs[C] //Proceedings of the 42nd Graphics Interface Conference. Waterloo: Canadian Human-Computer Communications Society, 2016: 167-174

[6] Cuenca E, Sallaberry A, Wang F Y, et al. MultiStream: a multiresolution streamgraph approach to explore hierarchical time series[J]. IEEE Transactions on Visualization and Computer Graphics, 2018, 24(12): 3160-3173

[7] Card S K, Mackinlay J D, Shneiderman B. Readings in information visualization: using vision to think[M]. San Francisco: Morgan Kaufmann Publishers Inc., 1999

[8] Harrower M, Brewer C A. ColorBrewer.org: an online tool for selecting colour schemes for maps[J]. The Cartographic Journal, 2003, 40(1): 27-37

[9] Gramazio C C, Laidlaw D H, Schloss K B. Colorgorical: creating discriminable and preferable color palettes for information visualization[J]. IEEE Transactions on Visualization and Computer Graphics, 2017, 23(1): 521-530

[10] Wang L J, Giesen J, McDonnell K T, et al. Color design for illustrative visualization[J]. IEEE Transactions on Visualization and Computer Graphics, 2008, 14(6): 1739-1754

[11] Lee S, Sips M, Seidel H P. Perceptually driven visibility optimization for categorical data visualization[J]. IEEE Transactions on Visualization and Computer Graphics, 2013, 19(10): 1746-1757

[12] Fang H, Walton S, Delahaye E, et al. Categorical colormap optimization with visualization case studies[J]. IEEE Transactions on Visualization and Computer Graphics, 2017, 23(1): 871-880

[13] Hurter C, Serrurier M, Alonso R, et al. An automatic generation of schematic maps to display flight routes for air traffic controllers: structure and color optimization[C] //Proceedings of the International Conference on Advanced Visual Interfaces. New York: ACM Press, 2010: 233-240

[14] Wang Y H, Chen X, Ge T, et al. Optimizing color assignment 
for perception of class separability in multiclass scatterplots[J]. IEEE Transactions on Visualization and Computer Graphics, 2019, 25(1): 820-829

[15] Kim H R, Yoo M J, Kang H, et al. Perceptually-based color assignment[J]. Computer Graphics Forum, 2014, 33(7): 309-318.

[16] Wu C B, Ding Y, Zhou X X, et al. A grid algorithm suitable for line and area feature label placement[J]. Environmental Earth Sciences, 2016, 75(20): 1368

[17] Imhof E. Positioning names on maps[J]. The American Cartographer, 1975, 2(2): 128-144

[18] Christensen J, Marks J, Shieber S. An empirical study of algorithms for point-feature label placement[J]. ACM Transactions on Graphics, 1995, 14(3): 203-232

[19] Meng Y, Zhang H, Liu M C, et al. Clutter-aware label layout[C] //Proceedings of the IEEE Pacific Visualization Symposium. Los Alamitos: IEEE Computer Society Press, 2015: $207-214$
[20] Ali K, Hartmann K, Strothotte T. Label layout for interactive 3D illustrations[J]. Journal of WSCG, 2005, 13(1-3): 1-8

[21] Yamamoto M, Camara G, Lorena L A N. Tabu search heuristic for point-feature cartographic label placement[J]. GeoInformatica, 2002, 6: 77-90

[22] Pokonieczny K, Borkowska S. Using artificial neural network for labelling polygon features in topographic maps[J]. GeoScape, 2019, 13(2): 125-131

[23] Gazzaniga M S. The cognitive neurosciences[M]. 4th ed. Cambridges: MIT Press, 2009

[24] Margolin R, Tal A, Zelnik-Manor L. What makes a patch distinct?[C] //Proceedings of the IEEE Conference on Computer Vision and Pattern Recognition. Los Alamitos: IEEE Computer Society Press, 2013: 1139-1146

[25] Cleveland W S, McGill M E, McGill R. The shape parameter of a two-variable graph[J]. Journal of the American Statistical Association, 1988, 83(402): 289-300 